\title{
Hamsa
}

Journal of Judaic and Islamic Studies

\section{Le nom propre berbère (modèle tachelhit) Réflexion générale sur ses caractéristiques de base}

\section{Lahcen Daaiif}

\section{(2) OpenEdition}

1 Journals

\section{Édition électronique}

URL : https://journals.openedition.org/hamsa/843

DOI : $10.4000 /$ hamsa. 843

ISSN : 2183-2633

Éditeur

CIDEHUS - Centro Interdisciplinar de História Culturas e Sociedades da Universidade de Évora

\section{Référence électronique}

Lahcen Daaiif, « Le nom propre berbère (modèle tachelhit) Réflexion générale sur ses caractéristiques de base », Hamsa [En ligne], 3 | 2017, mis en ligne le 31 mars 2017, consulté le 13 mai 2021. URL: http://journals.openedition.org/hamsa/843 ; DOI : https://doi.org/10.4000/hamsa.843

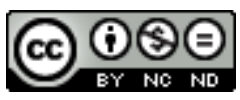

Hamsa est mise à disposition selon les termes de la Licence Creative Commons Attribution - Pas d'Utilisation Commerciale - Pas de Modification 4.0 International. 


\title{
Le nom propre berbère (modèle tachelhit) Réflexion générale sur ses caractéristiques de base*
}

\author{
Lahcen Daaïf \\ Chercheur associé à l'IRHT-CNRS, section arabe (Paris).
}

\begin{abstract}
Resumo :
Se bem que este estudo seja consagrado à onomástica berbere em geral, foca-se particularmente no nome próprio berbere e, especificamente no referente aos berberes Chleuhs do Anti-Atlas, que falam tachelhit. Neste sentido, favorece os laços de ordem onomástica entre, por um um lado a toponímia, a etnonímia e a antroponímia e, por outro, as fontes onomásticas, historiográficas e hagiográficas árabes. Enfim, este artigo procura igualmente, pôr em paralelo, com objetivos de análise comparativa, os componentes do nome próprio árabe assim como os do nome próprio berbere.
\end{abstract}

Palavras-chave: onomástica berbere, nome próprio berbere, tachelhit, laqab, nisba, kunya, onomástica árabo-islâmica.

\section{Résumé :}

Bien que cette étude soit consacrée à l'onomastique berbère en général, elle entend porter un intérêt particulier au nom propre berbère, et tout spécialement à celui porté par les berbères Chleuhs de l'Anti-Atlas, dont le parler est le tachelhit. A cet égard, elle favorise les liens d'ordre onomastique entre, d'une part la toponymie, l'ethnonymie et l'anthroponymie, et d'autre part, les sources onomastiques, historiographiques et hagiographiques arabes. Enfin, cet article s'efforce également de mettre en parallèle, à des fins évidentes d'analyse comparative, les composantes du nom propre arabe aussi bien que celles du nom propre berbère.

Mots-clés : Onomastique berbère, nom propre berbère, tachelhit, laqab, nisba, kunya, onomastique arabo-islamique.

Cette étude a pour objectif de construire une réflexion autour de la question générale de l'onomastique berbère, et plus particulièrement du nom propre berbère, dans un cadre de recherche académique, loin de toute visée idéologique à l'endroit de la berbérité ou de l'identité berbère. Pour ce faire, une mise en relation avec les organisations territoriales et communautaires, comme avec les systèmes de noms qui en découlent s'imposent. En effet, en raison de ses multiples variantes dialectales, la langue berbère ne se prête peut-être pas si facilement à ce type d'étude qui doit faire appel à plusieurs pans de spécialités en sciences humaines pour espérer parvenir à un résultat plus ou moins satisfaisant, sans qu'il soit pour autant définitif. II s'agira donc bien de l'onomastique berbère au sens propre de la discipline, mais où l'on favorisera une approche qui procédera autant que faire se peut à relier les noms toponymique, ethnonymique et anthroponymique à l'histoire, aux mœurs et à la sociologie de ce peuple qui s'est forgé un destin de résistance dont l'actualité politique et notamment culturelle continue encore à porter témoignage.

Dans cette étude on mettra davantage l'accent sur les liens évidents entre l'onomastique arabe et berbère en privilégiant l'aspect comparatif entre ces deux civilisations 
islamiques qui partagent une histoire commune au Maghreb. Les composantes structurelles du nom berbère, et surtout leurs différents fonds référentiels sont les principaux volets dont traite cet article. Aussi convient-il de souligner que les dénominations des objets et celles des lieux reposent sur des unités lexicales construites suivant une morphologie syntaxique tout à fait différente. Nous n'envisageons pas de nous y attarder de manière systématique, excepté bien évidemment dans des cas spécifiques que nous estimons d'un intérêt didactique. Toujours estil que l'on doit mettre en avant les éléments différentiels, le cas échéant, entre les dénominations anthroponymiques typiques d'une part, et celles d'ordre toponymique qui d'autre part n'obéissent pas aux mêmes modalités structurelles au point de vue linguistique.

\section{L'emprunt à l'arabe}

Avant d'aborder les divers fonds référentiels du nom propre berbère, bornons-nous en premier lieu à rappeler que celui-ci est constitué quasiment des mêmes composantes que le nom propre arabe (ism). On y trouve évidemment le nom de la personne, suivi de celui de son père, puis parfois celui de son grand-père; des noms reliés les uns aux autres par le terme ou qui signifie en berbère " fils de ", l'équivalent d'ibn en arabe. Vient ensuite clore cette succession de noms de personnes, le surnom (laqab), et parfois le nom de relation (nisba) qui est construit pour l'essentiel, avec le préfixe $a / i$ pour le masculin, et le préfixe ta et suffixe te pour le féminin ${ }^{1}$, comme nous l'illustrerons par des exemples plus loin.

On l'aura compris, l'une des composantes clés du nom propre arabe qu'est la kunya de relation filiale, comme dans l'arabe Abū Fulān (le père d'Untel) ${ }^{2}$, brille par son absence dans le nom berbère. Même si cette forme de kunya apparaît dans la chaîne onomastique de certains personnages berbères célèbres qui bénéficient de notices détaillées dans les sources hagiologiques et historiques ainsi que dans les recueils biographiques arabes, elle n'en reste pas moins une composante facultative. Certes, elle figure davantage dans les noms arabes de personnages historiques berbères, mais non de manière régulière et systématique comme cela est de rigueur dans les sources arabes. A titre d'exemple on se bornera à ces trois noms de figures berbères savantes qui portent une kunya : le traditionniste hāariǧite Abū al-Qāsim b. Sa'd al-Maknāsī ( $m$. fin $8^{\mathrm{e}}$, début $9^{\mathrm{e}}$ siècle), le juriste et cadi Abū 'Abd Allāh Muhammad b. Aswad alMadyūnī dont la date de mort est inconnue, et notamment le fameux juriste mālikite tunisien, 'Abd Allāh b. Muḥammad Ibn Abī Zayd al-Qayrawānī (m. 386/996) dont la kunya est Abū Muhammad $^{3}$. On notera que ces personnages sont mentionnés avec une nisba en lien avec leur lieu d'origine, ainsi que celle de Sūsī (originaire de Souss) pour Abū 'Alī Sālim b. Salāma (m. 589 ou 590/1193 ou 1194)4. Si d'autres noms de figures berbères intègrent quasi-systématiquement la kunya de relation filiale, cela laisse supposer qu'il était de bon aloi dans le milieu arabe savant que des Berbères portent une kunya, sans qu'elle fût nécessairement prise en compte dans le

\footnotetext{
* L'écriture tifinagh, dont la graphie et les règles orthographiques sont encore sujettes à de nombreux débats entre les spécialistes de la langue berbère, de même que la translittération par l'alphabet phonétique international sont délaissée expressément dans cette étude, au profit d'une translittération simplifiée des termes berbères (par ex., on usera de $g h$ au lieu de $y$ comme dans Aghyûl (âne), au lieu de Ayûl). Quant aux termes arabes, ils seront translittérés selon le système de la revue des arabisants: Arabica.

${ }^{1}$ Cette construction rejoint certaines formes d'annexion qui varient suivant les dialectes berbères, voir $S$. Chaker, Encyclopédie Berbère, Aix-en-Provence, Edisud, 1988, V, p. 686-695. On consultera entre autres Capitaine Justinard, Manuel de berbère marocain (Dialecte Chleuh), Paris, Librairie orientale et américaine, 1914, p. 5.

2 J. Sublet, Le voile du nom, Paris, PUF, 1991, p. 40-42.

${ }^{3}$ Cf. Anonyme, Mafāhir al-Barbar, éd. 'Abd al-Qādir Būbāya, Rabat, Dār Abī Raqrāq, 2005, p. 153-154.

${ }^{4}$ Anonyme, Mafāhir al-Barbar..., p. 165.
} 
milieu d'origine de ces personnages religieux, où régnait la langue berbère ${ }^{5}$. En revanche, les autres formes de kunya, notamment celle de prestige qui exprime une notion de bienfait ou l'idée de qualité morale ${ }^{6}$, propitiatoire ${ }^{7}$ ou métonymique, semble avoir la préférence des Berbères.

Du fait de l'islamisation des sociétés berbères au Moyen-âge, la plupart des noms berbères se trouvent être directement empruntés à l'arabe. A la tête de ce type de noms, vient tout naturellement comme partout dans le monde musulman, le nom du Prophète Muhammad (le loué) $^{8}$ ainsi que ses autres qualificatifs, tels Aḥmad (le plus loué) et Mub̧tār (l'élu) qui deviennent respectivement sous leurs formes berbères: Mohmmad/ Mhmed/Mhnd/Moh, $H$ Hâd et Mkhtâr. Suivent en termes de fréquence, notamment dans le milieu chleuh, une série de noms composés avec pour premier terme d'annexion le mot 'Abd (serviteur). C'est principalement 'Abd Allāh ${ }^{9}$ (serviteur de Dieu) qui vient en tête. Il s'agit du nom du père du Prophète, prononcé en berbère 'Bdullâh/'Bdllâh, ou sous sa forme contactée de 'Blla. Il est suivi de celui de 'Abd al-Raḥmān (le serviteur du Miséricordieux), berbérisé en 'Bderḥmân, un nom sudarabique porté par l'un des dix Compagnons du Prophète promis au Paradis. D'autres noms d'éminents Compagnons du Prophète sont tout aussi bien portés, dont ceux des deux califes bien-guidés: 'Umar (longue vie) qui se prononce quasiment de la même façon en berbère, 'Alī (noble, haut) contracté en 'î̀; et le nom de Mas'ūd (heureux, chanceux) contracté en Ms 'ûd. Les noms des deux petits-fils du Prophète complètent cette série de noms à connotation religieuse : al-Hasan (beau) et son diminutif al-Husayn qui se prononcent respectivement suivant les régions et les dialectes berbères soit Lhassan et Lhussein, ou sous une forme excessivement contractée : Lḥsn et Lhus. ${ }^{10}$

Pour ce qui est des noms féminins, l'emprunt se fait massivement au même registre onomastique arabo-islamique, à savoir celui de la famille du Prophète, à commencer par les noms de ses épouses et ses filles. Aussi vient-il en premier parmi les noms de ses épouses, celui de sa favorite, fille d'Abū Bakr premier calife, 'A' 'iša (vivante) prononcé en berbère 'Icha, suivi de celui de sa première épouse Hadīğa (prématurée à qui l'on souhaite une longue vie), prononcé tel quel en berbère Hadīğa, ou sous sa forme diminutive en arabe Hudayğ qui devient simplement Khadij. Des noms des filles du Prophète, le plus usité est celui de sa fille cadette Fāțima (sevrée ou dont le petit a été sevré) décliné sous ses diverses formes en arabe qui ont donné en berbère Fațma, Fadna, Fazma ${ }^{11}$. Curieusement, dans le parler tachelhit du moins, ce nom est suivi de celui de la fille aînée du Prophète, Ruqayya (la fine, la douce) prononcé Rqiyya

${ }^{5}$ Anonyme, Mafāhir al-Barbar..., p. 153-172. Cf presque toutes les notices biographiques figurant dans Ibn al-Zayyāt Abū Ya'qūb Yūsuf al-Tādilī, al-Tašawwuf ilā riğāl al-tașawwuf wa-ahbār Abī al-'Abbās alSabtī, $2^{\mathrm{e}}$ édition, Casablanca, Mațba'at al-Nağāḥ al-ğadīda, 1997.

${ }^{6} \mathrm{~J}$. Sublet, Le voile du nom, p. 46.

7 Dont la kunya d'Abū al-Futūḥ Buluqīn (Bologhine) b. Zīrī (m. 373/984), le fondateur de la dynastie berbère des Zirides, cf. entre autres, Anonyme, Mafāhir al-Barbar, p. 96.

${ }^{8}$ Sur les prénoms composés à base de Muhammad, on constate le même phénomène en Algérie, où ils sont devenus nombreux depuis le début du $3^{\mathrm{e}}$ millénaire, voir Farid Benramdane qui en propose I'illustration à travers plusieurs tableaux détaillés, $i d$. "De l'usage des bases anthroponymiques et des particules filationnelles (ben, bou, bent, moul, mohamed, el amine..) dans les prénoms algériens ", in Des noms et des noms... Anthroponymie et état civil en Algérie, éd. F. Benramdane, Oran, Edition CRASC, 2005, p. 81-96, p. 86-88.

${ }^{9} L^{\prime}$ usage abondant de prénoms composés avec 'Abd et son évolution depuis le début du $20^{\text {e }}$ siècle en Algérie mérite d'être mis en comparaison avec l'usage qui en est fait au Maroc, voir F. Benramdane, "De l'usage des bases anthroponymiques et des particules filationnelles ॥, p. 89-90.

${ }^{10}$ La plupart de ces noms ainsi que d'autres dont nous n'avons pas tenu compte ici pour des raisons de brièveté sont portés par des Chleuhs fréquentés par Capitaine Justiniard, voir id., Manuel de berbère marocain, p. V.

${ }^{11}$ Même remarque à faire à propos de prénoms composés à partir de Fāțima et de Hadīğa qui a connu un regain d'intérêt les deux dernières décennies, cf. F. Benramdane, «De l'usage des bases anthroponymiques et des particules filationnelles », p. 84-85. 
par suppression de la première voyelle entraînant la vocalisation en ' $i$ ' de la deuxième voyelle 'a' comme c'est souvent le cas devant un $y \bar{a}$ ' doublé, particulièrement quand il s'agit d'une forme diminutive.

Certains noms de Prophètes mentionnés dans le Coran sont davantage prisés que d'autres. Les plus souvent portés dans l'espace rural notamment, sont: Ibrāhīm/Abaham (Brahim ou contracté en Bihi), Ismāīl/Ismaël (Smaîin ou Smîn), Dāwud/David (Dâwd), Sulaymān/Salomon (Slîmân) Yaḥyā/Jean (Iḥyâ), Yūsuf (Yûsf) et dans une moindre mesure Mūsā/Moïse ${ }^{12}$ dont la prononciation ne subit aucune altération. Cet ordre de fréquence anthroponymique est abandonné de nos jours, de même que la forme berbérisée de ces noms traditionnels dont on ne trouve plus trace actuellement que dans les zones rurales, qu'il s'agisse des compagnes reculées ou des régions montagneuses escarpées. Mais puisque le processus d'arabisation des sociétés maghrébines n'a pas épargné l'onomastique berbère en général, l'on ne s'étonnera pas de voir que le nom propre arabe, présent autrefois sous sa forme berbérisée, est aujourd'hui transcrit et prononcé plus ou moins correctement en conformité à sa forme arabe d'origine. Ce changement au niveau de la prononciation des noms berbères d'origine arabe est la conséquence directe de la politique de ré-arabisation de l'anthroponymie personnelle quand bien même nous assisterions au cours de ces deux dernières décennies à un intérêt croissant pour le nom propre berbère, revendiqué comme une réaffirmation de l'identité berbère dans son originalité perdue. On voit bien que ces deux langues en cohabitation depuis des siècles, sont sciemment instrumentalisées dans un rapport de confrontation idéologique entre Berbères et Arabes, mais dans lequel prévalent comme enjeu identitaire primordiale, la révision des dénominations et la re-nomination à outrance ${ }^{13}$.

\section{Les noms de relation $(\text { nisba })^{14}$}

Est-il nécessaire de faire remarquer que, tout comme sa sœur arabe, la langue berbère se sert abondamment de noms de relation pour construire non seulement des anthroponymes, mais aussi des ethnonymes, bien que ce soit à une moindre échelle. Néanmoins, en comparaison avec l'arabe, pour former des noms de relation (nisba), le berbère tend à se reporter à une palette de références imaginaires, culturelles et naturelles qui semble bien moins riche et variée en termes du patrimoine consigné par écrit. En dépit de cette quantité ténue de référents qu'on y déplore, on peut aisément relever que les noms formés comme une nisba n'en sont pas moins présents que dans l'arabe.

Comme les exemples abondent en ce sens, on doit mettre l'accent de préférence sur les noms de relation avérés dans les sources historiques, hélas toutes rédigées en arabe, et dans les récentes études d'anthropologie berbère en arabe et en langues occidentales. L'exemple incontournable en l'occurrence est le toponyme de Huwwāra d'où est tiré la célèbre nisba en arabe, Huwwārī, répandue dans tout le Maghreb. Ce nom de relation qui a pour pendant en berbère Ahuwwari au masculin, Tahuwwarit au féminin, est souvent mentionné dans les sources arabes sous sa forme arabe, ainsi qu'en fait foi entre autres le nom de l'historien maghrébin Mu'min b. Yumar al-Huwwārī, qui fut l'une des sources principales d'al-Bakrī pour la rédaction

12 Le nom de Mûsâ ainsi que 'î reviennent souvent dans les récits et contes rapportés par Capitaine Justiniard, dans son Manuel de berbère marocain, voir p. 83, 84, 85

${ }^{13} \mathrm{~S}$ 'agissant de l'arabe, S. Chaker parle de pression forte sur le berbère, id., Encyclopédie berbère, Aix-enProvence, Edisud, 2008, XXVIII-XXIX, p. 4360.

${ }^{14}$ Sur la nisba, voir J. Sublet, Le voile du nom, p. 95-113. 
de son histoire du Maghreb ${ }^{15}$. Signifiant grosso modo " suzerains ", "dominants ${ }^{16}$, Huwwāra ${ }^{17}$ est un vocable générique donné à l'origine aux populations berbères de la région tripolitaine, plus exactement celles peuplant la région de Fezzan en Libye ${ }^{18}$. Mais il y a des toponymes pour cantons et villages berbères qui peuvent aussi bien faire office de référents à la formation d'un nom de relation berbère. Ainsi le nom de Achtûk, propagé surtout par le chanteur traditionnel (raïs) et poète S'îd Achtouk, désigne une personne originaire de la région de Chtouka; de même le nom de Atanân, Tatanânt au féminin, pour les personnes originaires de la commune rurale de Ida-Outanan, ces deux régions étant situées dans la pleine de Souss au Sud du Maroc. Située juste au voisinage nord de Souss, la région de Hạhạa (l'une des plus grandes tribus de la branche Masmouda $)^{19}$ sert de référent au nom de relation de Ihihî, au féminin Tihihit, un nom porté par de nombreux chanteurs et chanteuses populaires adulés par le public berbère marocain. Parmi les noms formés sur ce même schème, on ne perdra pas de vue celui de Azûlay qui peut signifier étymologiquement aussi bien l'homme aux beaux yeux qu'aux yeux clairs, et renvoyer en même temps à un nom de relation désignant un habitant de la ville de Tazoulayte, située au centre-est du Maroc. On signalera au passage que ce nom est d'actualité tant au Maroc qu'en France, puisqu'il est porté de nos jours par de grandes familles juives occupant des responsabilités politiques dans les deux pays ${ }^{20}$.

Parmi les grandes familles zénètes (Iznatn) ${ }^{21}$ qui régnaient au Maghreb central (Algérie), les sources arabes relatent les exploits de l'une d'elles, connue sous le nom de Beni Yfrn, de la descendance du roi Yfrn ${ }^{22}$. Constituée de plusieurs tribus, cette famille, d'après Ibn Khaldoun, $s^{\prime}$ était détournée du sunnisme pour embrasser le Kharidjisme ${ }^{23}$. Etant le pluriel de ifri, ce nom, qui veut dire grotte ou caverne, serait par ailleurs à l'origine du nom d'une petite ville touristique marocaine : Ifrane (les grottes).

Il est des noms de cette structure qui sont tombés dans l'oubli depuis longtemps, parce qu'ils proviennent généralement d'un nom de relation (nisba) lui-même désuet. En fait partie par exemple, le nom de relation de Gellidassen ou Gueldacen mentionné ainsi dans les sources arabes ${ }^{24}$ d'où la difficulté d'une translittération exacte. II renvoie en réalité, au toponyme

${ }^{15}$ Voir al-Bakrī qui le mentionne dans son al-Masālik wa-l-mamālik, éd., A. P. Van Leeuven et A. Ferre, Tunis, al-Dār al-'arabiyya li-I-kitāb, 1992, II, p. 851, n 1422, p. 762, nº 1278.

${ }^{16}$ M. Cast, " H 61. Huwwâra, Hoouara, Houara, Hawwra ", Encyclopédie Berbère, Aix-en-Provence, Edisud, 2000, XXIII, p. 3513. Par contre S. Chaker ne reconnaît aucune signification confirmée à ce mot provenant de la racine HWR/Ihawwaren, qui serait à l'origine de l'ethnique actuelle Ahaggar/Ihaggaren, voir id., "La langue berbère à travers l'onomastique médiévale : El-Bakri », Revue de l'Occident musulman et de la Méditerranée 35 (1983), p. 127-144, p. 130.

17 Nom donné à l'une des grandes tribus constitutives de la branche berbère de Baranes, selon l'auteur anonyme de Mafāhir al-barbar, p. 158.

${ }^{18}$ M. Cast, «H 61. Huwwâra, Hoouara, Houara, Hawwra».

${ }^{19}$ Anonyme, Mafāhir al-Barbar, p. 172. Voir aussi Ibn Haldūn, Tārīh Ibn Haldūn (Kitāb al-'Ibar wa-dīwān al-mubtada' wa-l-habar), éd., Halīl Šahāta, Beyrouth, Dār al-Fikr, 1431/2000, VI, p. 299.

${ }^{20}$ Dont Andrey Azoulay conseiller du roi du Maroc, Mohammed VI, et sa fille Audrey Azoulay actuellement ministre de la culture dans le gouvernement de François Hollande.

${ }^{21}$ Sur le sens supposé initial de ce terme, voir les suggestions linguistiques (faraḍiyyāt) de 'Alī Șidqī Azaykū, « Al-Ta'wīl al-nasabī (al-ǧīnyālūḡī) li-tārīh Šamāl Ifrīqyā hal yumkinu taǧāwuzuhu ? » (Peut-on dépasser I'interprétation généalogique de l'histoire de l'Afrique du Nord ?), Mağallat kulliyyat al-'ulūm wa-l-ādāb al-insāniyya, Rabat, 1989-1990, p. 9-34, p. 29.

${ }^{22}$ Elle est souvent mentionnée dans al-Bakrī, al-Masālik wa-l-mamālik, voir par ex., II, p. 827, $\mathrm{n}^{\circ} 1381, \mathrm{p}$. 829, n 1384. Voir Ibn Haldūn, Kitāb al-'lbar, VII, p. 11, 13, 15.

${ }^{23}$ Sur leur conversion au Kharidjisme voir Ibn Haldūn, Kitāb al-'Ibar, VII, p. 17, sur Bani Yfrn voir plus spécialement p. 15-38 ; C. Agabi, «I 34. Ifren (Beni) 》, Encyclopédie Berbère, XXIV, p. 3657 ; voir Robert Montagne, Le Berbère et le Makhzen dans le Sud du Maroc, Paris, Félix Alcan, 1930, p. 29, 60.

${ }^{24}$ Il faut rappeler que ce nom ainsi que d'autres que nous signalerons plus loin, sont tirés du livre d'Abū 'Ubayd Allāh al-Bakrī (m. 478/1094), al-Masālik wa-l-mamālik, la partie consacrée au Maghreb, Description de l'Afrique septentrionale, trad. Mac Guckin De Slane, Paris, Imprimerie impériale, 1859, par 
lelidassen, qui désigne une ville située entre Ténès et Achîr (Algérie) ${ }^{25}$, qui signifie plus vraisemblablement " leur roi » suivant la structure syntagmatique de la jonction du nom à un affixe personnel, en l'occurrence assen (leur), comme le soulignent P. Morizot ${ }^{26}$ et S. Chaker ${ }^{27}$.

\section{Le fonds référentiel animalier}

Les noms d'animaux s'avèrent également un riche vivier anthroponymique pour les Berbères, qui semblent bénéficier davantage à l'anthroponyme masculine que féminine. Bien que moins porté dans certaines régions que d'autres, Ifis (hyène), qui apparaît dans les contes berbères comme l'une des bêtes féroces les plus redoutées dans l'inconscient collectif berbère ${ }^{28}$, est délaissé pour d'autres noms d'animaux beaucoup plus fascinants pour leur qualité de courage auxquels ils sont généralement associés dans l'imaginaire berbère. Ifis est toutefois donné principalement comme surnom ou sobriquet aux personnes dont on voudrait signaler des particularités négatives telles la férocité et la bestialité par lesquelles elles se trouvent ainsi identifiés à l'hyène.

Le nom de certains animaux s'avère ainsi convenir à des hommes dans l'espoir d'être imprégnés de leur bravoure et leur endurance. Les plus souvent considérés pour ces qualités parmi les fauves qui peuplaient évidemment les plaines et les montagnes du territoire berbère, peuvent d'être ramenés grosso modo au quatre suivants que sont naturellement le lion, le lionceau, le tigre ou la panthère, et le guépard. II s'agit respectivement en berbère de $1 \mathrm{zm}^{29}$, $\operatorname{Aghilas}^{30}$, principalement répandu en Kabylie, $A k s i^{31}$ et $A m a y a s$, ce dernier étant plutôt répandu au Maroc ${ }^{32}$. D'autres animaux qui relèvent de cette catégorie dont les origines sont attestées dans les dessins rupestres d'avant la période caméline, sont encore d'usage patronymique. On citera parmi les principaux noms qui se réfèrent à cette catégorie, Ar'm (chameau) -il s'agit plus précisément dans ces régions de dromadaire- ${ }^{33}$, Odad (mouflon) qui symbolise la résistance farouche dans les montagnes rocheuses, et dans une moindre mesure Arksim (caracal).

Aux oiseaux aussi, ont été empruntés des noms destinés aussi bien aux hommes qu'aux femmes dont les plus courants sont, au féminin : Tanina, qui renvoie « à une sorte d'oiseau

les soins de $\mathrm{S}$. Chaker, dans son article, "La langue berbère à travers l’onomastique médiévale : El-Bakri ", p. 127-144. Désormais, le texte arabe d'al-Bakrī est plus indiqué, vu qu'il est disponible en édition critique avec introduction en français et un riche index des noms propres et des lieux, voir id, al-Masālik wa-lmamālik.

25 S. Chaker, Linguistique berbère, études de syntaxe et de diachronie, Paris-Louvain, Editions Peeters, 1995, p. 154 ; id., " La langue berbère à travers l'onomastique médiévale : El-Bakri », p. 130.

${ }^{26}$ P. Morizot, «I 25. lelidassen », Encyclopédie Berbère, XXIV, p. 3639.

27 S. Chaker, "La langue berbère à travers l'onomastique médiévale : El-Bakri », p. 130. Cette forme ancienne n'est plus d'usage dans aucun dialecte berbère parlé de nos jours. Voir les remarques de Aḥmad al-Tawfĩq, l'éditeur d'al-Tādilī, al-Tašawwuf, p. 115, note 100, n 16 : « Abū 'Uțmān Sa'd b. Mīmūnasn alRağrāğī », Mimûnassen (celui qui leur porte bonheur) étant construit suivant le même schème que Gellidassen; voir aussi id., p. 227, note 80 : « Abū Muḥammad Ǧllidāsn b. Isḥāq ", p. 261, n 111 : « Abū Mahdī Wīn Slāmt Ǧllidāsn » etc.

${ }^{28}$ H. Camps-Fabrer, " H 63. Hyène ॥, Encyclopédie Berbère, XXIII, p. 3555 ; Emile Laoust, Contes berbères du Maroc, Paris, Larose, 1949, v. 2, p. XX.

${ }^{29}$ D'où Izmrasen (le puissant) autre nom berbère. Sur cet animal dans les contes populaires en tachelhit, voir E. Laoust, Contes berbères du Maroc, v. 2, p. XVIII.

${ }^{30}$ E. Laoust, Contes berbères du Maroc, v. 2, p. XIX.

${ }^{31}$ Dont probablement le nom de Kusayla b. Lzem (m. 67/686), le chef militaire berbère qui opposa une longue résistance à la conquête arabe, et fut tué par Qays b. Zuhayr al-Balawī, cf. Ibn Haldūn, Tārīh, VI, p. 142 , p. 193-194.

32 M. A. Haddadou, Recueil de prénoms berbères, Alger, 2003, p. 15.

${ }^{33}$ H. Lhote, "Camelin ou période cameline », Encyclopédie Berbère, VI, p. 935-936. 
mythique célèbre pour sa beauté et sa noblesse ", et Timilla ou Tamilla (tourterelle) ${ }^{34}$; et au masculin : Aylâl (oiseau), Atbîr (colombe) ${ }^{35}$ et surtout Igidr (aigle) qui symbolise l'indépendance majestueuse ${ }^{36}$.

Un nom peut provenir également d'une image qui sous-tend l'imaginaire collectif. Elle peut symboliser des qualités positives auxquelles s'identifiaient un clan, une tribu ou une famille, telles que la résistance, la consistance ou la solidité. Même si d'ordinaire une qualité de ce type est associée à un objet inanimé, comme par exemple un mur de soutènement qui se dit en berbère igherm, elle n'en est pas moins le nom toponymique d'une région célèbre de Souss située au sud-ouest ${ }^{37}$ de Taroudant dans l'Anti-Atlas. Comme souvent, ce genre de toponyme manque rarement de se transformer en nom de famille. Au Maroc, en effet, Igherm représente l'un des patronymes réputés, porté avec fierté par de grandes familles. Conjointement au courage qu'inspirent certains animaux, d'autres qualités du même registre peuvent servir de noms à une personne censée les incarner, ou sur laquelle tout simplement on les projette. Au fil du temps, quelques noms dérivés de ces qualités parviennent à se perpétuer en tant que nom de famille dans la descendance de l'ancêtre éponyme. On le constate par exemple, avec le nom de Yarniyân qui renvoie à l'idée de victoire sur l'ennemi ${ }^{38}$, ou avec le nom de Badis, autre catégorie de noms hautement appréciés par les Berbères, pour les qualités de résistance, de courage et de combativité qu'il dénote. Ce dernier nom est attesté dans tout le Maghreb depuis déjà l'antiquité ainsi qu'en témoignent les inscriptions libyques où il figure sous ses formes originelles de Bedis, Badisn qui signifient se tenir debout, se dresser et être prêt à se défendre.

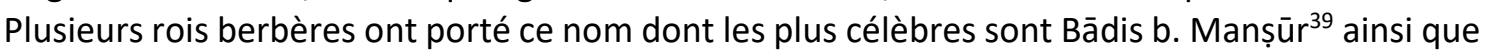
des figures marquantes de l'histoire du Maghreb, tel le réformateur algérien 'Abd al-Ḥamīd Ibn Bādis (m. 1940).

\section{Des noms de bon présage}

Comme dans l'onomastique de la plupart des langues, le berbère accorde une place prépondérante aux noms d'affects abstraits qui expriment des notions telles que le bonheur, la joie, la prospérité, la fortune et la chance etc. A vrai dire, c'est dans cette classe de noms que le berbère tire en substance l'une des portions les plus riches et les plus variées de son anthroponymie. En témoigne l'un des plus célèbres noms de la nomenclature des figures historiques du Maghreb, celle du fondateur du mouvement almohade, al-Mahdī lbn Tūmart ( $\mathrm{m}$. $524 / 1130$ ), Tūmart signifiant en berbère " bonheur, joie ${ }^{40}$. Pas moins célèbre cet autre nom de Yaghmurasen qui fut porté par plusieurs souverains berbères. Il évoque le même sens, puisqu'il est de la même racine que le premier, Yaghmur $^{41}$. Des noms kabyles pour filles, tels que Tafsut (printemps), Tala (fontaine), Tazrurt (la belle) ou Tlaten (elle les subjugue), Titrit

\footnotetext{
34 J. M. Dallet, Dictionnaire Kabyle-Français, SELAF, Paris, 1982, II, p. 489a ; M. A. Haddadou, Recueil de prénoms berbères, p. 17.

35 Dont le nom du dévot et ascète Abū 'Alī Atbīr b. Yirzğān al-Rağrāğī qui vécut en retraite dans une grotte, cf. al-Tādilī, al-Tašawwuf, p. 418-419, n² 243.

${ }^{36}$ Voir sur la représentation de l'aigle dans la société berbère de Souss, cf. Aki'o Nakano, Ethnographical Texts in Moroccan Berber (3) (Dialect of Anti-atlas), Studia Berber (III), Tokyo, Institute for the Study of Languages and Cultures of Asia and Africa, 1998, p. 50.

${ }^{37}$ A. Zaguef, "Igherm", Encyclopédie Berbère, XXIV, p. 3670-3671.

${ }^{38} \mathrm{~S}$. Chaker, "La langue berbère à travers l'onomastique médiévale : El-Bakri », p. 139, nº 82.

${ }^{39}$ Voir sur l'Etat de Bādīs b. al-Manșūr et les guerres qu'il livrait aux Zénètes, Ibn Haldūn, Kitāb al- 'lbar, VI, p. 208-209; sur son successeur, son fils, al-Mu'izz b. Bādīs, ibid., VI, p. 209-211.

${ }^{40} \mathrm{~S}$. Chaker, "La langue berbère à travers l'onomastique médiévale : El-Bakri », p. 139, $\mathrm{n}^{\circ} 86$.

${ }^{41} \mathrm{~S}$. Chaker, "La langue berbère à travers l'onomastique médiévale : El-Bakri », p. 139, $\mathrm{n}^{\circ} 82$. Nom porté par le saint Abū 'Alī Yaġmūr b. Hālid, voir al-Tādilī, al-Tašawwuf, p. 286, n 129. Voir sur les personnages qui répondent à ce nom Ibn Haldūn, Kitāb al-'Ibar, VII, p. 215 (Yaġmūrasn b. Salāma), VI, p. 191 (Yaġmūrasn b. Abī Samḥa), VII, p. 471-473 (Yaġmūrasn b. Muḥammad al-Sālifī).
} 
(étoile) pour ne citer que les plus souvent donnés aux filles, ne laissent aucun doute quant à leur dimension propitiatoire. Ils visent en effet, à s'attirer les faveurs du sort pour que les filles dénommées ainsi soient désirables, bien portantes, et qu'elles deviennent surtout de futures mères fécondes. Le nom arabe Sa'îd (heureux), est fort présent dans la nomenclature patronymique berbère noble ou d'extraction supérieure sous sa forme contractée de $S^{\prime} i ̂ d$ au masculin, et $S^{\prime}$ diyya au féminin (dérivé de Sa'diyya ${ }^{42}$ et non de Sa'īda). De même le prénom masculin As'dî, Tas'dît au féminin, connu également sous sa forme francisée Tassadit ${ }^{43}$ est une épithète qui désigne respectivement le chanceux et la chanceuse. Or, dans les milieux populaires ou d'extraction inférieure, c'est plutôt le nom arabe Mas'ūd contracté en Ms'ûd et Ms'ûda au féminin, qui est d'usage. S'insèrent dans cette catégorie le nom de Mimûn (de l'arabe Maymūn), dont le nom du juriste et saint soufi Abū 'Uțmān Mimûnassen al-Rağrāḡ ${ }^{44}$, et son féminin Tamaymûnt (la chanceuse) ${ }^{45}$, ainsi que le nom composé Bû-unnûr, plus connu sous la forme populaire de Sidi Bennûr 'Abd Allāh b. Wakrīs al-Dukkālī ${ }^{46}$, ou celui d'un autre personnage saint de Marrakech Abū Luqmān Yirzǧān, en berbère Îrzgân, sing. Îrzg (la bonne étoile) ${ }^{47}$. On mentionnera pour finir le nom de Iddr, Tiddr, vivant/vivante, l'équivalent de 'Ayyāš/'A' 'iša en arabe, qui serait porté dans le passé sous forme composée, avec le suffixe assen, Iddrassen, comme dans le nom de Mūsā b. Yiddrassen al-Ḥallāğ ${ }^{48}$.

\section{Les traits et particularités physiques}

Dans ce domaine aussi on notera que la langue berbère ne fait pas exception par rapport aux autres langues, ou du moins par rapport à la plupart d'entre elles. Elle rejoint dans le domaine des patronymes en lien avec le corps et ses déficiences, les langues chamito-sémitiques auxquelles elle appartient. En ce qui concerne les noms qui se réfèrent aux traits, aux particularités et essentiellement aux tares physiques, ils y sont légion. Pour en donner un aperçu significatif, on se focalisera sur les noms de cette nature largement encore en usage dans le Maghreb, et tout spécialement au Maroc. Bien évidemment, tout comme dans l'arabe, ces noms sont portés comme des patronymes, issus des surnoms qui ont été suscités autrefois par les particularités physiques des ancêtres. Nous en voulons pour preuve Aderghal (le malvoyant) ${ }^{49}$, un nom qui subsiste partout dans toute l'aire géographique berbère, et même en Europe. Plus exactement on le rencontre au Sud de la France où il serait apparu depuis le $19^{\mathrm{e}}$ siècle $\mathrm{e}^{50}$, $\mathrm{d}^{\prime}$ où la probabilité qu'il soit plus ancien encore en Espagne. Portant aussi sur une tare de la vue, Aziwâl $\left.\right|^{51}$, le bigleux, est souvent attribué comme surnom, devenu patronyme de nombreuses familles, tout comme d'ailleurs Azrwâl, qui désigne une personne aux yeux bleus ou verts, les deux couleurs étant à l'origine désignées par le même mot comme dans la langue arabe. En raison de cette particularité physique qui leur est commune, les membres d'une tribu entière

42 Peut-être est-ce en référence au nom de nisba de la nourrice du Prophète, Ḥalīma al-Sa'diyya, personnage vulgarisé par la fameuse Hamziyya du poète berbère de Șanhāğa, al-Būșīī, Muḥammad b. Sa'ĩd (m. 696/1295).

${ }^{43}$ Ce prénom est largement diffusé sous cette forme au féminin en France, principalement dans les milieux berbères kabyles.

${ }^{44}$ Al-Tādilī, al-Tašawwuf, p. 115.

${ }^{45}$ Al-Tādilī, al-Tašawwuf, p. 234, n 88 : « Abū Wakīl Maymūn b. Tāmaymūnt ».

${ }^{46}$ Al-Tādilī, al-Tašawwuf, p. 130, n²2.

${ }^{47}$ Al-Tādilī, al-Tašawwuf, p. 232, n 86.

${ }^{48}$ Un des disciples du grand soufi Abū Madyan al-Ġawt, cf. al-Tādilī, al-Tašawwuf, p. 330, n 166.

${ }^{49}$ S. Chaker, Linguistique berbère, p. 14-15.

${ }^{50}$ Voir le site http://www.genealogie.com/nom-de-famille/ADERGHAL.html, consulté le 3/3/2016.

51 II peut être donné comme toponyme, comme c'est le cas avec l'un des sommets de la région des Seksawa au Haut-Atlas marocain, cf. J. Berque, Structures sociales du Haut-Atlas, Paris, PUF, 1955, p. 43, 44. 
dans la région de Souss sont ainsi appelés Izrwalen, ou Beni Zerwa $\left.\right|^{52}$ le singulier étant Azrwâl. On rappellera à ce propos Ḥassān b. Zarwāl, le personnage mentionné par Ibn Khaldoun comme I'un des commandants rapprochés du premier émir omeyyade d'al-Andalus 'Abd al-Rahmān alDāhil « l'immigré » (m. 172/788) ${ }^{53}$, qui est une figure militaire de la tribu de Maġīla. Cependant, si le toponyme de Tazerwalt ${ }^{54}$, qui a abrité Sidi Ḥmâd ou Mûsâ, le saint le plus éminent de Souss ${ }^{55}$, est tiré de la même racine, il aurait pour étymologie plutôt l'abondance et la richesse naturelle dont témoigne la verdure de cette région par contraste avec les terres alentour.

D'autres noms doivent être pris en compte dans cette classe d'onomastique qui constitue l'une des sources de référence intarissable de la patronymie berbère. Dans le cadre restreint de cette étude, on insistera sur les quelques noms qui nous paraissent les plus récurrents. Il s'agit bien évidemment des noms qui décrivent des défaillances physiques aux conséquences manifestes dans le comportement physique et moral de l'individu. Abugad

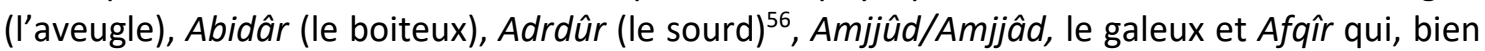
que dérivant de l'arabe où il signifie pauvre, désigne en berbère le vieillard-la vieillesse s'inscrit ainsi dans le registre des tares physiques ${ }^{57}$-, représentent les noms les plus usités de cette classe d'onomastique. Une région entière peut être désignée par un nom issu d'une caractéristique physique qui serait spécialement propre à sa population, comme en témoigne le nom de relation arabisé d'al-Ǧazūlī, ${ }^{58}$ que porte entre autres ${ }^{59}$, le savant soufi Muhammad b. Sulaymān al-Ǧazūlī (m. 870/1465) connu pour son recueil des prières rituelles dédiées au Prophète Muhammad, Dalā'il al-hayrā et le théologien fondateur du mouvement almoravide 'Abd Allāh b. Yāsīne alǦazūlī (m. 451/1059) ${ }^{60}$. Ainsi, contrairement à ce que suggère Azayku (ce nom qu'il s'est donné, signifiant ancien, ancestral, atteste d'un attachement idéologique à la culture berbère) dans ses suppositions révisionnistes ${ }^{61}$ difficilement cautionnables, ce nom est à l'origine une épithète, au 52 Dans les sources arabes, comme les nomme Ibn Haldūn dans son Histoire, voir R. Montagne, Le Berbère
et le Makhzen dans le Sud du Maroc, p. 209.
53 Ibn Haldūn, Kitāb al-'Ibar, VI, p. 164.
${ }^{54}$ Sur l'histoire de Tazerwalt, voir C. Justinard, Un petit royaume berbère, Le Tazeroualt. Un saint berbère
Sidi Ahmad ou Moussa, Paris, Librairie orientale et américaine \& G.P. Maisonneuve, 1954, et les travaux
dirigés par Paul Pascon, La maison d'Iligh et l'histoire sociale du Tazerwalt, Rabat, Société des Marocains
des Éditeurs Réunis, 1984. Sur ses mythes et récits voir, Paulette Galand-Pernet, Littératures berbères, des
voix, des lettres, Paris, PUF, 1998, p. 103, 203 ; Robert Montagne, Le Berbère et le Makhzen dans le Sud
du Maroc, p. 203 et passim.
55 . C. Justinard, Un petit royaume berbère, Le Tazeroualt. Un saint berbère Sidi Ahmad ou Moussa, p. 1833.

${ }^{56}$ Ces trois qualités qui se trouvent être coraniques laissent supposer une similitude avec l'arabe dans cette classe d'onomastique. Voir sur les deux premiers mots Capitaine Justinard, Manuel de berbère marocain (Dialecte Chleuh), Paris, Librairie orientale et américaine, 1914, p. 114.

57 S. Chaker, "La langue berbère à travers l'onomastique médiévale : El-Bakri », p. 134. Au lieu d'Ismg comme patronyme, les Kabyles usent d'Akli/iklan, esclave, comme prénom, appellation qui désignait les Berbères d'origine servile, issus principalement de la Boucle du Niger, cf. Francis Manzano « Berbères, berbérité : noms, territoires, identités ", Cahiers de sociolinguistique, Rennes, PUR, 2006, p. 175-214, p. 188.

${ }^{58}$ Région à laquelle Muḥammad al-Muḩtār al-Sūsī a consacré son ouvrage de relation intitulé justement, Hilāl Ğazūla, Tétouan (Maroc), al-Mațba'a al-mahdiyya, s.d., 4 vol.

${ }^{59}$ Comme par exemple Abū Muḥammad Irzğān al-Ǧazūlī (7e siècle de l’hégire), al-Tādilī, al-Tašawwuf, p. $267, \mathrm{n}^{\circ} 114$.

${ }^{60}$ Voir entre autres, N. Levtzion, “'Abd Allāh b. Yāsīn and the Almoravids », in J. R. Willis (éd.), Studies in West African Islamic History, I, The Cultivators of Islam, Londres, 1979, p. 78-112; C. Justinard, Un petit royaume berbère, Le Tazeroualt, p. 82.

${ }^{61}$ Pour 'Alī Șidqī Azāykū, il y a une autre lecture probable de ce mot, qui laisserait entendre que les Igzzulen seraient les descendants des Gétules, puisque ces derniers étaient dispersés partout dans le Maghreb en raison de leur nomadisme. De là, explique-t-il, la signification exacte de ce mot, par contraction de kus (souk) et ulli (le bétail), serait plutôt « les éleveurs de bétails », cf. id., " Al-Ta’wīl al-nasabī (al-ǧīnyālūḡī) 
pluriel, donnée aux habitants d'une région de Souss occidentale (Maroc), en raison de leur petite taille, d'où Igzzula, Igzzulen, singulier Agzzûl62. Pareillement avec le nom Amezyan qui signifie petit en âge ou cadet, ainsi que son antonyme Ameqran, grand ou aîné, tous deux subsistent encore dans l'usage des noms partout dans le Maghreb ${ }^{63}$. Si la taille du corps compte comme référence dans la formation des noms de personnes, la couleur de la peau est tout aussi importante pour qualifier une personne et la désigner facilement. Le blanc et le noir sont sans conteste les deux couleurs les plus usitées dans la formation des noms, Oumlil, le blanc et Asggan/Asuqqi pour le noir. Il y a deux autres couleurs qui jouissent d'un usage proche : le rouge et le jaune. Cependant, ils ne se réfèrent pas systématiquement à la couleur de la peau de l'ancêtre éponyme, dans la mesure où la toponymie est susceptible de jouer le rôle central de référent pour une personne qui y est originaire. Ainsi, par exemple, le nom de personne Azggâgh peut se référer au toponyme d'Adrâr Azggâgh/Azkkâgh, qui veut dire la Montagne Rouge, une des palmeraies du Sud de Figuig au Maroc ${ }^{64}$.

Néanmoins, on ne perdra pas de vue que ce sont plutôt les noms composés qui constituent la majeure partie de cette classe d'onomastique. II s'agit de noms formés de préposition de conjonction « bu » (celui ou l'homme à, en arabe $\underline{d} \bar{u}$, au féminin $\underline{d} \bar{a} t$ ) qui permet de relier la personne dénommée à sa particularité physique, à travers un nom substantif qui désigne une partie, un membre ou un organe du corps humain. Toute une nomenclature de noms de ce type qui s'est progressivement constituée au fil du temps, permet maintenant des choix infinis de noms portés aujourd'hui encore y compris par des familles juives maghrébines. Pour ne tenir compte que des plus communément usités, il nous suffira de mentionner ces patronymes dont regorgent les annuaires téléphoniques des pays du Maghreb, voire de la France, d'Espagne et même d'Italie. Mais en raison du caractère polyréférentiel dont nous avons croisé quelques exemples au travers d'une pluralité de noms ou de signifiants pour un seul et même référent, il ne nous serait possible d'en passer en revue que les plus usuels. Aussi a-t-on bû-ohlig, celui au gros ventre ; bû-ofus, le manchot ; bu-owajjâj ${ }^{65}$, I'homme à lunettes ; bi-ifaddn, I'homme costaud (littéralement celui aux genoux vigoureux); bû-agayyu, celui à l'énorme tête ; bî-imzgân, celui avec de grandes oreilles; bî-ifnza, celui aux gros orteils ; bi-idodân, celui aux doigts géants ; bû-unfûr, celui à la lèvre énorme ou fourchue etc. Bien que de structure similaire à première vue, certains noms se réfèrent plutôt à une localité comme le montre le nom de bûabûd, celui au nombril particulier, dans la région de Souss qui découle sans aucun doute d'un ancien patronyme passé à la postérité en devenant un toponyme ${ }^{66}$.

li-tārīh Šamāl Ifrīqyā hal yumkinu taǧāwuzuhu ? ", p. 30-31. Voir aussi sur les Yaġmūr, Ibn Haldūn, ibid., V, p. 415,416 ; VI, p. 35,81 ; VII, p. 74, 178, 632.

${ }^{62} \mathrm{~S}$. Chaker, Linguistique berbère, p. 154. Quelques membres de cette tribu, qui étaient installés à Cadix, ont habité un village qui porte encore leur nom : Alcalá de los Gazules, voir M. Ghouirgate, «Asmās : le plat emblématique des Almohades ", Arabica 62 (2015), p. 144, note 75. Sur les Igzzulen qui seraient des Berbères originaires de Sanhadja, venus du Sahara vers le Souss, et sur leur rapport aux tribus avoisinantes, voir R. Montagne, Le Berbère et le Makhzen dans le Sud du Maroc, p. 193-211.

63 M. A. Haddadou, Recueil de prénoms berbères, p. 14. Voir sur les formes adjectivales et les diminutifs de ces deux mots M. Kossmann, The Arabic Influence on Northern Berber, Leyde, Brill, 2013, p. 286-287. 64 Voir Mohamed Yeou, "A propos de quelques toponymes à Figuig et sa région ", Asinag 6 (2011), p. 129-147, p. 135-136.

${ }^{65}$ A ne pas confondre avec le nom de Wâggâg, épithète qui désigne une personne très douée en sciences religieuses, d'où le nom de Sidi Wāğğāğ b. Zllū al-Lamțī, juriste mālikite et fondateur de l'une des premières medersas de la région de Souss, située à Aglou près de Tiznit, cf. al-Tādilī, al-Tašawwuf, p. 89$91, n^{\circ} 5$.

${ }^{66}$ Cette localité est souvent mentionnée par M. Muhntār al-Sūsī dans ses œuvres, voir à titre d'exemple, Hilāl Ğazūla, Tétouan, s.d., I, p. 45, 46 et passim. 


\section{Prestige et rang social}

La fonction distinguée tout aussi bien que le rang social ou le prestige dont jouit un individu représente une source presque aussi riche et prisée que le fonds référentiel des particularités physiques pour la formation des noms en berbère. En effet, qu'il s'agisse d'un rang social supérieur ou inférieur, les deux niveaux ont donné lieu à une multitude de noms patronymiques qui parfois peuvent faire fonction d'indicateur sur l'ancien statut social, culturel ou politique de l'ancêtre d'une famille, d'une tribu, voire d'une ethnie. L'on comprend dès lors que le statut bas d'Ismg, ou de $s m g o^{67}$, esclave ou nègre, ou d'Aderfi, l'affranchi (l'équivalent de mawlā en arabe), soit porté comme nom tout comme le statut élevé d'Agllid, roi, d'Amghâr ${ }^{68}$, le chef de la tribu qui règle les litiges entre ses membres ou d'Agurrâm ${ }^{69}$ qui tire son prestige d'un ancêtre saint ou de sa descendance du Prophète auquel tout le monde témoigne du respect.

Dans cette même perspective, les métiers exercés par les uns et les autres traduisent leur appartenance sociale et partant, reflètent leur rang et leur représentativité au sein de leur groupe ethnique ou social. D'aucuns se trouvent à jamais liés aux métiers qu'ils ont exercé ou aux fonctions religieuses qu'ils ont occupé eu égard au nom qu'ils en ont hérité. Ainsi par exemple, Amzîl, le forgeron ${ }^{70}$, qui désignait jadis le métier de l'ancêtre, est passé comme nom de famille à sa descendance, de même que $L m$ ûddn, Muezzin ${ }^{71}$ (de l'arabe al-mu'addin) dont la fonction religieuse est d'appeler les fidèles à la prière du haut du minaret de la mosquée du village. En effet, on est impressionné par le nombre de familles qui portent ces deux noms patronymiques au Maghreb. Jusqu'à une date récente, dans les milieux urbains à majorité berbère, et de nos jours encore dans certains milieux ruraux berbères du Maroc, l'épithète de hāğğg, en berbère l'hâjjj, qualifiant toute personne ayant accompli le pèlerinage à La Mecque, est accolée si systématiquement au nom qu'elle finit parfois par en supplanter la composante essentielle qu'est le nom personnel (le prénom). L'hâajj devient ainsi un nom de prestige en place et lieu du nom propre ${ }^{72}$. C'est aussi à cette catégorie de noms que l'on doit la persistance de la kunya propitiatoire dans les noms patronymiques berbères que nous avons illustrés par quelques exemples auparavant. II convient d'y ajouter les deux kunya-s d'un des plus célèbres

\footnotetext{
${ }^{67}$ Al-Bakrī mentionne le nom du traditionniste hāriǧite de la branche șufrite, Abū al-Qāsim Smǧū (pour Smg்ū) de Meknès (al-Maknāsī), dans al-Masālik wa-l-mamālik, II, p. 838, $\mathrm{n}^{\circ}$ 1400. C'est le même personnage mentionné dans Anonyme, Mafāhuir al-Barbar, sans ce qualificatif de Smg்ū à la page 153, et en entier à la page 140 : Abū al-Qāsim Smġū b. Wāsūl (cf. supra, note 3). Ce même personnage apparaît chez Ibn Haldūn, Kitāb al-'Ibar, VI, p. 172, d'une orthographe légèrement différente, Smqū ou Smkū. On notera cependant que dans le milieu kabyle, contrairement aux noms de smgo ou ismg attribués comme surnom, c'est Akli (esclave) qui prévaut nom seulement comme nom, mais également comme prénom. Cf. S. Chaker, "La langue berbère à travers l'onomastique médiévale : El-Bakri », p. 133.

68 Encore en usage de nos jours, Amġār (Amghâr) était également porté par plusieurs personnages religieux mentionnés dans le recueil biographique des saints d'al-Tādilī, al-Tašawwuf, dont au moins trois occurrences : p. 209, $n^{\circ} 75 ;$ p. $231, n^{\circ} 87 ;$ p. 426, $n^{\circ} 254$.

${ }^{69}$ Voir Aki'o Nakano, Ethnographical Texts in Moroccan Berber (3) (Dialect of Anti-atlas), Studia Berber (III), Sur la fonction précise et le prestige dont jouissait Amghâr p. 66 ; et le respect dû par tous à Agurrâm de par son ascendance noble du point de vue religieux « žždd-nnsn imqqurn iga y-l'ar n-nnbi (Leur grand ancêtre est de la famille du Prophète [Muhammad] », p. 67.

${ }^{70}$ Sur le métier d'Amzîl, le forgeron, nécessaire et incontournable pour un ou plusieurs village, cf. Aki'o Nakano, Ethnographical Texts in Moroccan Berber (2) (Dialect of Anti-atlas), Studia Berber (II), Tokyo, Institute for the Study of Languages and Cultures of Asia and Africa, 1995, p. 2.

${ }^{71}$ Sur la fonction du Muezzin, cf. Aki'o Nakano, Ethnographical Texts in Moroccan Berber (3), p. 61 ; et sur les anecdotes qui y sont associées, voir Capitaine Justiniard, Manuel de berbère marocain, p. 41-42.

72 « kudnna-d ikka lḥižž, ar-as aqqran mddn, " wa lhažž " niy " wa lhažža " (quand il a accompli le pèlerinage à La Mecque, les gens l'appellent "ô Ihâajj" ou "ô lhâjjja ") ", cf. Aki'o Nakano, Ethnographical Texts in Moroccan Berber (2), p. 96.
} 
saints de l'hagiographie marocaine, Sidi Bûy'zzâ (Abū Ya'zā) et Yilannûr ${ }^{73}$. Toutes les deux sont composées de deux substantifs arabes, 'izz (gloire) et nūr (lumière), précédés des préfixes bû et yila, équivalents en arabe de $\underline{d} \bar{u}$ dont nous avons précédemment traité, désignant ainsi le saint comme étant en possession de la gloire et de lumières spirituelles.

\section{Conclusion}

Dans l'onomastique berbère, le nom propre est sans doute porteur de nombreuses indications qui le rapprochent, par certaines caractéristiques structurelles et formelles, non seulement du nom arabe, mais aussi du fonds référentiel commun à plusieurs civilisations environnantes de langues diverses. Sans être aussi diversifié et varié dans ses composantes de base que le nom arabe auquel on s'est employé à le comparer, le nom berbère, du moins au Maroc, principalement sous sa forme arabisée telle qu'elle est consignée dans les sources arabes, peut suffire à décliner l'essentiel de l'identité de celui qui le porte. En revanche, du fait de la concision qui le caractérise dans l'usage qui en est fait dans la vie réelle dans certains milieux ruraux et conservateurs, on constate que, malgré son emprunt indubitable à l'arabe, il n'est susceptible de nous renseigner qu'au niveau anthropologique, voire sociologique, sur des informations qui varient considérablement en fonction des lieux géographiques et des aires culturelles qui plus est aux délimitations incertaines.

73 Al-Tādilī, al-Tašawwuf, p. 213- 222, n 77 ; Victorien Loubignac, « Un saint berbère, Moulay Bou'azza. Histoire et légende ", Hespéris 31 (1944), p. 15-34. 